\title{
CERTAIN RADIALLY DILATED CONVOLUTION AND ITS APPLICATION
}

\author{
Jungsoo RheE
}

\begin{abstract}
Using some interesting convolution, we find kernels recovering the given function $f$. By a slight change of this convolution, we obtain an identity filter related to the Fourier series in the discrete time domain. We also introduce some techniques to decompose an impulse into several dilated pieces in the discrete domain. The detail examples deal with specific constructions of those decompositions. Also we obtain localized moving averages from a decomposition of an impulse to make hybrid Bollinger bands, that might give various strategies for stock traders.
\end{abstract}

\section{Introduction}

Let us start with the definition of the Fourier transform of $f$ : for $f \in L^{1}(R)$, the Fourier transform of $f$ is defined by

$$
\hat{f}(w)=\int_{-\infty}^{\infty} f(t) e^{-2 \pi i w t} d t, w \in R
$$

or $\hat{f}(t)=\frac{1}{\sqrt{2 \pi}} \int_{-\infty}^{\infty} f(x) e^{-i t x} d x$ for $t \in R$. Here the $L^{p}\left(R^{n}\right)$ is the spaces, $1 \leq p<\infty$, of all measurable functions $f$ such that

$$
\|f\|_{p}=\left(\int_{R^{n}}|f(x)|^{p} d x\right)^{\frac{1}{p}}<\infty .
$$

Let $f$ and $g$ be measurable functions on $R^{n}$. The convolution of $f$ and $g$ is the function $f * g$ defined by $f * g(x)=\int_{R} f(x-y) g(y) d y$ for all $x \in R^{n}$ such that the integral exists.

Received February 25, 2010. Accepted March 8, 2010.

2000 Mathematics Subject Classification. 44A35, 42B99

Key words and phrases. convolution, kernels, identity filter, Fourier series, Bollinger bandss 
It is known that an approximate identity recovers the given function using a convolution operator and a dilation of certain kernel(see[F]). Therefore let us consider a convolution (see also [St]):

$$
\int_{0}^{\infty} \int_{R^{n}} f(x-y) s^{n-1} \phi(s y) d y d s .
$$

Then this convolution is well defined for a continuous function $f$ if $\phi$ is a proper kernel. Also it has special properties recovering the given function and many useful applications. We deal with these properties in the next section.

\section{Few theorems of radially dilated convolutions}

Theorem 2.1. Let $f \in L^{1}\left(R^{n}\right)$ be a continuous function. Suppose that $\phi$ is a continuous bounded radial function ( by abusing notation, let us write $\phi(r)=\phi(x)$, if $|x|=r)$ with the compact support, $[0, M]$, for some $M>0$. If $\int_{0}^{M} s^{n-1} \phi(s) d s=0, n=1,2, \ldots$, we obtain

$$
\text { (1) } \cdots \int_{0}^{\infty} \int_{R^{n}} f(x-y) s^{n-1} \phi(s y) d y d s=c f(x),
$$

for each $x \in R^{n}$, when $c=\int_{R^{n}} \int_{0}^{1} s^{n-1} \phi(s y) d s d y$.

Proof. We put $K(y)=\int_{0}^{1} s^{n-1} \phi(s y) d s$ and $K_{m}(y)=m^{n} K(m y)$ for $m>0$. Because $\int_{0}^{M} s^{n-1} \phi(s) d s=0$, we get $K_{m}(y)=0$ for all $y \notin \bar{B}_{0}\left(\frac{M}{m}\right)$, the closure of the open ball of radius $\frac{M}{m}$ centered at 0 . In fact, we find

$$
\begin{aligned}
K_{m}(y) & =\int_{0}^{m} r^{n-1} \phi(r y) d r \\
& =\frac{1}{|y|^{n}} \int_{0}^{m|y|} s^{n-1} \phi\left(s \frac{y}{|y|}\right) d s \\
& =\frac{1}{|y|^{n}} \int_{0}^{M} s^{n-1} \phi(s) d s=0, \text { by the homogeneity of } \phi .
\end{aligned}
$$

Let $\epsilon>0$ be given and let $x$ be any fixed element in $R^{n}$. Since $f$ is uniformly continuous on $\bar{B}_{x}(1)=\left\{y \in R^{n}:\|y-x\| \leq 1\right\}$, a compact subset containing $x$, we get $|f(x-y)-f(x)|<\epsilon$ whenever $\|y\|<\delta$ for some $\delta>0$. 
Then we find

$$
\begin{aligned}
& \left|\int_{R^{n}} f(x-y) K_{m}(y) d y-c f(x)\right| \\
\leq & \int_{\bar{B}_{0}\left(\frac{M}{m}\right)}|f(x-y)-f(x)|\left|K_{m}(y)\right| d y \\
\leq & \epsilon \int_{\bar{B}_{0}\left(\frac{M}{m}\right)}\left|K_{m}(y)\right| d y \\
& \left(\text { for large m satisfying }|y|<\frac{M}{m}<\delta\right) \\
\leq & \epsilon \int_{\bar{B}_{0}\left(\frac{M}{m}\right)} m^{n} \int_{0}^{1} r^{n-1}|\phi(r m y)| d r d y \\
\leq & \epsilon \sigma\left(S^{n-1}\right) \frac{N M^{n}}{n^{2}},
\end{aligned}
$$

where $\sigma\left(S^{n-1}\right)$ is the spherical measure of the n-dimensional sphere $S^{n-1}$ and $\phi$ is bounded by $N$ for some $N>0$.

Since we have

$$
\int_{0}^{\infty} \int_{R^{n}} f(x-y) s^{n-1} \phi(s y) d y d s=\lim _{m \rightarrow \infty} \int_{R^{n}} f(x-y) K_{m}(y) d y,
$$

we prove that (1) holds for each $x$ at which $f$ is continuous.

Let $S\left(R^{n}\right)$ be the set of Schwartz-class functions which consists of all $f \in C^{\infty}\left(R^{n}\right)$ satisfying $\sup _{x}\left|x^{\gamma} \partial^{\alpha} f(x)\right|<\infty$. Here $\alpha=\left(\alpha_{1}, \ldots, \alpha_{n}\right), \gamma=$ $\left(\gamma_{1}, \ldots, \gamma_{n}\right)$ and $x^{\gamma}=x^{\gamma_{1}} \cdots x^{\gamma_{n}}, \partial^{\alpha}=\left(\frac{\partial}{\partial x_{1}}\right) \cdots\left(\frac{\partial}{\partial x_{n}}\right)$. Then the above dilated convolution defined on $S\left(R^{n}\right)$ reminds us of the recovering theorem in the wavelet analysis(see $[\mathrm{C}],[\mathrm{D}]$ and $[\mathrm{K}])$.

Theorem 2.2. Let $f \in S\left(R^{n}\right)$. Suppose that $\phi$ is a bounded radial function and suppose that $\phi$ is an integrable function with an admissibility condition satisfying $\int_{0}^{\infty} \frac{|\hat{\phi}(r)|}{r} d r<\infty$. Then, for $c_{\phi}=\int_{0}^{\infty} \frac{\hat{\phi}(r)}{r} d r$, we have

$$
\int_{0}^{\infty} \int_{R^{n}} f(x-y) r^{n-1} \phi(r y) d y d r=c_{\phi} f(x),
$$

for all $x \in R^{n}$. 
Moreover, if we put $\Psi_{0}(x)=\int_{0}^{1} r^{n-1} \phi(r x) d r, \Psi_{1}(x)=\int_{1}^{2} r^{n-1} \phi(r x) d r$ and $\Psi_{k}(x)=2^{n(k-1)} \Psi_{1}\left(2^{k-1} x\right), k=1,2, \ldots$, we obtain

$$
\sum_{k=0}^{\infty} f * \Psi_{k}(x)=c_{\phi} f(x) .
$$

Proof. We put $f * \phi(r \cdot)(x)=\int_{R^{n}} f(x-y) \phi(r y) d y$. Since $f \in S\left(R^{n}\right)$, we have $\|f * \phi(r \cdot)\|_{\infty} \leq\|\phi\|_{\infty}\|f\|_{1}$ and $\|f * \phi(r \cdot)\|_{1} \leq \frac{1}{r^{n}}\|\phi\|_{1}\|f\|_{1}$, for each $r>0$. Since $\hat{f} \in S\left(R^{n}\right)$ and $(f * \phi(r \cdot))^{\wedge}(x)=\frac{1}{r^{n}} \hat{\phi}\left(\frac{x}{r}\right) \hat{f}(x)$, we have $\|\hat{f}(\cdot) \hat{\phi}(\dot{\bar{r}})\|_{1} \leq\|\hat{\phi}\|_{\infty}\|\hat{f}\|_{1}$, for each $r>0$. Also $f * \phi(r \cdot)$ is continuous on $R^{n}$ for each $r>0$. Therefore $f * \phi(r \cdot)(x)=\int_{R^{n}} \hat{f}(y) \hat{\phi}\left(\frac{y}{r}\right) \frac{1}{r^{n}} e^{2 \pi i x \cdot y} d y$, for all $x \in R^{n}$, by the Fourier invrsion theorem.

So, for any $\epsilon>0$ and $m>0$, we obtain

$$
\begin{aligned}
\int_{\epsilon}^{m} \int_{R^{n}} f(x-y) r^{n-1} \phi(r y) d y d r & =\int_{\epsilon}^{m} \int_{R^{n}} \hat{f}(y) \hat{\phi}\left(\frac{y}{r}\right) \frac{1}{r} e^{2 \pi i x \cdot y} d y d r \\
& =\int_{R^{n}} \hat{f}(y) \int_{\epsilon}^{m} \hat{\phi}\left(\frac{y}{r}\right) \frac{1}{r} d r e^{2 \pi i x \cdot y} d y \\
& \leq \int_{R^{n}}|\hat{f}(y)| \int_{0}^{\infty}\left|\hat{\phi}\left(\frac{y}{r}\right)\right| \frac{d r}{r} d y \\
& \leq \int_{R^{n}}|\hat{f}(y)| \int_{0}^{\infty}\left|\hat{\phi}\left(\frac{y}{s|y|}\right)\right| \frac{d s}{s} d y \\
& \leq \int_{0}^{\infty} \frac{|\hat{\phi}(r)|}{r} d r|| \hat{f} \|_{1},
\end{aligned}
$$

by the Fubini's theorem and the homogeneity of $\phi$.

By taking $\epsilon \rightarrow \infty$ and $m \rightarrow \infty$, we have, by the similar process in the above calculations,

$$
\begin{aligned}
\int_{0}^{\infty} \int_{R^{n}} f(x-y) r^{n-1} \phi(r y) d y d r & =\int_{R^{n}} \hat{f}(y) \int_{0}^{\infty} \hat{\phi}\left(\frac{y}{r}\right) \frac{1}{r} d r e^{2 \pi i x \cdot y} d y \\
& =\int_{R^{n}} \hat{f}(y) \int_{0}^{\infty} \hat{\phi}\left(\frac{1}{s}\right) \frac{d s}{s} e^{2 \pi i x \cdot y} d y \\
& =\int_{R^{n}} \hat{f}(y) \int_{0}^{\infty} \frac{\hat{\phi}(r)}{r} d r e^{2 \pi i x \cdot y} d y \\
& =c_{\phi} f(x)
\end{aligned}
$$


for all $x \in R^{n}$.

The other part of the theorem is just a series representation of the previous one. Thus we prove our theorem.

Now we know that the dilated convolution is well defined when $f$ is continuous by Theorem 2.1 and Theorem 2.2. Even though $f$ is not a continuous function, the dilated convolution might have finite values almost everywhere.

In a different way we describe in Theorem 2.1, we consider a Dirac typed convolution from the dilated convolution that related to an approximate identity.

Theorem 2.3. We put $\phi(x)=\frac{1}{\pi} \int_{0}^{1} g(t) \cos t x d t$, where $g$ is bounded and $g$ satisfies Dini's condition ( i.e., $\frac{g(t)}{t}$ is integrable on $\left.(0,1)\right)$ and $\phi(0)=1$. Let $\Psi_{0}(x)=\int_{0}^{1} \phi(s x) d s$ and $\Psi_{n}(x)=n \Psi_{0}(n x)$. We set $\Psi(x)=\lim _{n \rightarrow \infty} \Psi_{n}(x)$. Suppose that $\int_{R} \Psi_{0}(x) d x=1$. Then $\Psi$ is a Dirac delta function .

Moreover, if $\left|\Psi_{0}(x)\right| \leq C(1+|x|)^{-1-\epsilon}$ for some $C, \epsilon>0$, we have

$$
\int_{0}^{\infty} \int_{R} f(x-y) \phi(s y) d y d s=\lim _{n \rightarrow \infty} f * \Psi_{n}(x)=f(x), f \in L^{1}(R),
$$

for almost all $x \in R$.

Proof. Since $\phi(0)=1$, we get $\Psi(0)=\infty$.

If $x \neq 0$, we have

$$
\begin{aligned}
\Psi(x) & =\lim _{n \rightarrow \infty} \Psi_{n}(x) \\
& =\lim _{n \rightarrow \infty} \frac{1}{\pi} \int_{0}^{1} g(t) \int_{0}^{n} \cos t r x d r d t \\
& =\lim _{n \rightarrow \infty} \frac{1}{\pi} \int_{0}^{1} g(t) \frac{\sin n t x}{t x} d t=0 \text { by Dini's condition. }
\end{aligned}
$$

Also $\lim _{n \rightarrow \infty} \int_{R} \Psi_{n}(x) d x=\int_{R} \Psi_{0}(x) d x=1$. Thus $\Psi$ is a Dirac function. For the proof of remaining part, see Theorem 8.15 in $[\mathrm{F}]$. 


\section{Few theorems related to radially dilated convolutions in the discrete time domain}

From Theorem 2.3, we will construct some discrete kernel that recover the given sampled data.

We put $g(t)=t h(t)$ in Theorem 2.3, where $H$ is an antiderivative of $h$ such that $H^{\prime}(t)=h(t)$ and $H(1)=0$. Then we have

$$
\begin{aligned}
\Psi_{0}(x) & =\frac{1}{\pi} \int_{0}^{1} h(t) \frac{\sin t x}{x} d t \\
& =\frac{1}{\pi} \int_{0}^{1}(-H(t)) \cos t x d t .
\end{aligned}
$$

Hence Theorem 2.3 gives that

$$
\lim _{n \rightarrow \infty} \frac{1}{\pi} \int_{R} \int_{0}^{1} f(x-y)(-H(t)) n \cos n t y d t d y=f(x),
$$

for almost all $x \in R$, when $g$ satisfies the same hypothesis in Theorem 2.3 .

Thus, we can construct lots of Fejer type of kernels from $\Psi_{0}$. As an example, if we put $-H(t)=1-t$, then we have $\Psi_{0}(x)=\frac{1}{\pi} \int_{0}^{1}(1-$ t) $\cos t x d t$.

By a simple calculation, we obtain

$$
\begin{aligned}
\Psi_{n}(x) & =\frac{1}{2 \pi} \int_{-n}^{n}\left(1-\frac{|t|}{n}\right) e^{-i t x} d t \\
& =\frac{2 \sin ^{2} \frac{n}{2} x}{\pi n x^{2}} .
\end{aligned}
$$

In $[R]$, we discretize $\Psi_{2 \pi n}(x)=\frac{\sin ^{2} \pi n x}{\pi^{2} n x^{2}}$ by a proper manner. Then we obtain some interesting kernel

$$
K_{m, n}(x)=\frac{n}{m}\left[1+2 \sum_{k=1}^{m-1}\left(1-\frac{k}{m}\right) \cos 2 \pi n \frac{k}{m} x\right] .
$$

This is a reminiscent of the Fejer kernel, $F_{n}(x)=\frac{\sin ^{2} \frac{n}{2} x}{2 \pi n \sin ^{2} \frac{x}{2}}$. It is known that $f * K_{n}$ and $f * K_{m, n}$ converges to $f$ uniformly on a compact subset $F$ of $R$ where it is continuous(see[R]).

Thus, we consider the following a discrete typed convolution that recover the given sampled data: 
Theorem 3.1. Let $f\left(\frac{j}{T}\right)$ be the sampled data with the sampling period $\frac{1}{T}$ where $j=0,1,2, . ., p-1$. Then we can recover data $f\left(\frac{l}{T}\right)$ from the kernel $\frac{1}{n} K_{m, n}(l), m>n p, n=1,2, \ldots$, by the following discrete convolution :

$$
f\left(\frac{l}{T}\right)=\sum_{j=0}^{p-1} f\left(\frac{j}{T}\right) \frac{1}{n} K_{m, n}(l-j), l=0,1,2, \ldots, p-1 .
$$

Furthermore, we have

$$
f\left(\frac{l}{T}\right)=\frac{1}{m}\left[a_{m, n}^{0}+2 \sum_{k=1}^{m-1}\left(1-\frac{k}{m}\right)\left(a_{m, n}^{k} \cos 2 \pi n \frac{k}{m} l+b_{m, n}^{k} \sin 2 \pi n \frac{k}{m} l\right)\right],
$$

where $a_{m, n}^{k}=\sum_{j=0}^{p-1} f\left(\frac{j}{T}\right) \cos 2 \pi n \frac{k}{m} j, k=0,1,2, \ldots, m-1$

and $b_{m, n}^{k}=\sum_{j=0}^{p-1} f\left(\frac{j}{T}\right) \sin 2 \pi n \frac{k}{m} j, k=1,2, \ldots, m-1$.

Proof. By a simple calculation, we have

$$
\frac{1}{n} K_{m, n}(l)=2 \pi \frac{1}{m} F_{m}\left(2 \pi \frac{n}{m} l\right)=\frac{1}{m^{2}} \frac{\sin ^{2} \pi n l}{\sin ^{2} \frac{\pi n}{m} l}
$$

It is easy to check $\frac{1}{m^{2}} \frac{\sin ^{2} \pi n(l-j)}{\sin ^{2} \frac{\pi n}{m}(l-j)}=\delta(l-j)$ where $\delta(l-j)=1$ for $l=j$ and 0 for $l \neq j, j=0,1,2, \ldots, p-1$ : In fact, since $\lim _{x \rightarrow 0} \frac{1}{n} K_{m, n}(x)=1$, singularity at 0 is removable, i.e., $\frac{1}{n} K_{m, n}(0)=1$. Thus we have

$$
\sum_{j=0}^{p-1} f\left(\frac{j}{T}\right) \frac{1}{n} K_{m, n}(l-j)=\sum_{j=0}^{p-1} f\left(\frac{j}{T}\right) \delta(l-j)=f\left(\frac{l}{T}\right) .
$$

The remaining part of the theorem comes from the direct calculation of $K_{m, n}$. Thus, this completes our proof.

Remark 3.2. As we have seen in the previous theorem, we introduce a discrete type of nonharmonic Fourier series : Let us consider $N$ number of data, $f(0), f(1), \ldots, f(N-1)$. Then we define the discrete Fourier transform as

$$
F\left(\frac{k}{m}\right)=\sum_{l=0}^{N-1} f(l) e^{-i 2 \pi \frac{k}{m} l}, m>2 N
$$


Taking a modified inverse transform, we obtain

$$
\begin{aligned}
\sum_{k=-(m-1)}^{m-1} \frac{1}{m}\left(1-\frac{|k|}{m}\right) F\left(\frac{k}{m}\right) e^{i 2 \pi \frac{k}{m} n} & =\sum_{l=0}^{N-1} f(l) K_{m, 1}(n-l) \\
& =f(n), \text { for } 0 \leq n<N
\end{aligned}
$$

Now we construct a discrete type of the dilated convolution. It is a discrete typed version of $\sum_{k=0}^{\infty} f * \Psi_{k}(x)=c_{\phi} f(x)$ in Theorem 2.2. In this case, the dyadic dilation plays an important role to decompose an impulse.

Theorem 3.3. Suppose that $\phi$ is a bounded even function that has a support in $[-1,1]$ of $R$. Also we suppose that $\int_{0}^{1} \phi(x) d x=0$ and $\phi(0)=1$. We set $\Phi_{0}(x)=\int_{0}^{1} \phi(r x) d r, \Phi_{1}(x)=\int_{1}^{2} \phi(r x) d r$ and $\Phi_{k}(x)=$ $2^{k-1} \Phi_{1}\left(2^{k-1} x\right), k=1,2,3, \ldots$. Let $T$ be a sampling period. Let $f\left(\frac{l}{T}\right)$ be a given sampled data where $l=\ldots .-m,-(m-1), \ldots,-1,0,1,2,3, \ldots p,(p+$ $1), \ldots,(p+M), \ldots$ Then we recover $f\left(\frac{l}{T}\right), l=1,2, \ldots, p$, by the following procedure:

$$
f\left(\frac{l}{T}\right)=\sum_{k=0}^{n} \sum_{j=l-m}^{l+M} f\left(\frac{j}{T}\right) \frac{1}{2^{n}} \Phi_{k}\left(\frac{l-j}{2^{n}}\right), n=0,1,2, \ldots
$$

Proof. By the definition of $\Phi_{0}$, we prove easily that $\Phi_{0}(n)$ is the unit sample which is called the impulse, i.e., $\Phi_{0}(0)=1$ and $\Phi_{0}(n)=0$ for each $n= \pm 1, \pm 2, \ldots$. Let us decompose this impulse $\Phi_{0}(n)$ using the dilation property. In fact, we have naturally $\Phi_{1}(x)=2 \Phi_{0}(2 x)-\Phi_{0}(x)$ by a simple calculation. The dilation property gives us that $\sum_{k=0}^{n} \Phi_{k}(x)=$ $2^{n} \Phi_{0}\left(2^{n} x\right)$. Hence $\sum_{k=0}^{n} \frac{1}{2^{n}} \Phi_{k}\left(\frac{i}{2^{n}}\right)=0$ for each nonzero integer $i$ and 1 for $i=0$, since $\Phi_{0}(n)$ is the impulse. Thus we prove our theorem.

Remark 3.4. We get a different type of identity filter recovering sampled data from Theorem 3.3, compared to the Fejer typed kernel $K_{m, n}$ of Theorem 3.1. For more information about filters, see [L] and $[\mathrm{O}]$. As we have expected, the expression in Theorem 3.3 is a kind of sum of localized moving averages of data $f\left(\frac{l}{T}\right)$.

Now we construct two types of $\Phi_{0}(n)$. 
Example 3.5. with bounded support for $\Phi_{0}$. Now let $\phi(x)=$ $(-1) \chi_{\left[-1,-\frac{1}{2}\right)}(x)+\chi_{\left[-\frac{1}{2}, \frac{1}{2}\right]}(x)+(-1) \chi_{\left(\frac{1}{2}, 1\right]}(x)$. Then we get $\Phi_{0}(x)=1$ on $\left[-\frac{1}{2}, \frac{1}{2}\right]$, and $\Phi_{0}(x)=-1+\frac{1}{x}$ on $\left(\frac{1}{2}, 1\right]$. Also we have $\Phi_{0}(x)=-1-\frac{1}{x}$ on $\left[-1,-\frac{1}{2}\right)$ and $\Phi_{0}(x)=0$ for all $|x|>1$.

Let $n=5, m=4$ and $M=0$. Since we have $\Phi_{0}\left(\frac{i}{32}\right)=1$ for each $i=0, \pm 1, \ldots, \pm 15$, we get the first projection,

$$
\sum_{j=l-4}^{l} f\left(\frac{j}{T}\right) \frac{1}{2^{n}} \Phi_{0}\left(\frac{l-j}{2^{n}}\right)=\frac{1}{2^{5}} \sum_{j=0}^{4} f(l-j), T=1 .
$$

This is the scaled(modified) moving average of 5 data.

If $\Phi_{0}$ doesn't have bounded support, what happen? We want to find such an impulse $\Phi_{0}(n)$ induced by $\Phi_{0}$.

\section{Example 3.6. with unbounded support for $\Phi_{0}$.}

Suppose that $\Phi_{0}(x)=\int_{0}^{1} g(t) \cos 2 \pi t x d t$ where $g$ is a bounded function on $(-1,1)$ with $\Phi_{0}(0)=\int_{0}^{1} g(t) d t=1$ and suppose that $\Phi_{1}(x)=$ $2 \Phi_{0}(2 x)-\Phi_{0}(x)$. If $\Phi_{0}(k)=0$ for all nonzero integer $k$, then we can recover $f\left(\frac{l}{T}\right)$ by a decomposition of the unit sample $\Phi_{0}(n)$ like Theorem 3.3. There are many examples satisfying the property of $\Phi_{0}(n)$.

If $g(t)=2 t$ or $g(t)=2(1-t)$, then $\Phi_{0}(k)=0$ for all nonzero integer $k$ by a direct calculation.

When we choose $g(t)=1$, we have $\Phi_{0}(x)=\frac{\sin 2 \pi x}{2 \pi x}$. Thus $\Phi_{0}(0)=1$ because singularity at $x=0$ is removable and $\Phi_{0}(k)=0$ for all nonzero integer $k$. Hence we can write $f\left(\frac{l}{T}\right)$ as

$$
f\left(\frac{l}{T}\right)=\sum_{j=1}^{2^{n}} f\left(\frac{j}{T}\right) \frac{\sin 2 \pi(l-j)}{2 \pi(l-j)}, l=1,2, \ldots, 2^{n},
$$

which is the discrete-time representation of Shannon-Kotelnikov sampling theorem.

There are two different type of $\Phi_{0}$. One has a bounded support and the other has unbounded support. Actually $\Phi_{0}(n)$ is an impulse in the discrete time domain. Thus Theorem 3.3 describes how to decompose $\Phi_{0}(n)$ using dilation property. .

\section{Applications in DSP(Digital signal Processing) : Hybrid Bollinger Bands}


From decompositions of the unit sample, we have lots of identity filters. Since these filters are similar to sum of localized moving averages, we suggest to construct hybrid Bollinger bands.

Bollinger bands are well-known in stock markets as a popular technical analysis tool, that was introduced by John Bollinger in the early 1980 s(see $[\mathrm{B}]$ ).

Bollinger bands consist of a set of three curves :

Middle Bollinger band being 20-day simple moving average, Upper Bollinger band being Middle Bollinger band +2 ( 20-day standard deviation),

Lower Bollenger band being Middle Bollinger band - 2 ( 20-day standard deviation).

Because the standard deviation is a measure of volatility, the interval between the upper and lower bands and middle band is determined by volatility of stock prices. When the market becomes more volatile, the bands widen and the bands become narrow during less volatile period. Also stock data is not normally distributed. Thus, instead of finding about $95 \%$ of data inside the bands, we find less data. To improve the probability of data in Bollinger bands, a weighted moving average of Black-Scholes stock price model was used in [LHZ].

In our paper, replacing simple moving average by localized moving average filter, we suggest to make hybrid Bollinger bands.

20 -day moving average is defined by $\operatorname{mav}(l)=\frac{1}{20} \sum_{j=l-19}^{l} f(j), l=$ $1,2, \ldots, p$ for sampled data $f(j)$. Now we put middle band as

$$
m b(l)=\sum_{k=0}^{4} \sum_{j=l-19}^{l} f(j) \frac{1}{2^{5}} \Phi_{k}\left(\frac{l-j}{2^{5}}\right),
$$

where $\Phi_{0}$ is the given one in the first part of Example 3.5. Also we use the same moving standard deviation derived from $\operatorname{mav}(l)$ as

$$
\sigma(l)=\left(\sum_{j=l-19}^{l} \frac{1}{20}(f(j)-\operatorname{mav}(j))^{2}\right)^{\frac{1}{2}} .
$$

Then we get the upper hybrid Bollinger band as $u p(l)=m b(l)+2 \sigma(l)$ and the lower hybrid Bollinger band as $l o(l)=m b(l)-2 \sigma(l), l=$ $1,2, \ldots$, respectively.

We draw hybrid Bollinger bands for Naver's daily closing stock price from August,4th, 2009 to February, 22th, 2010. 


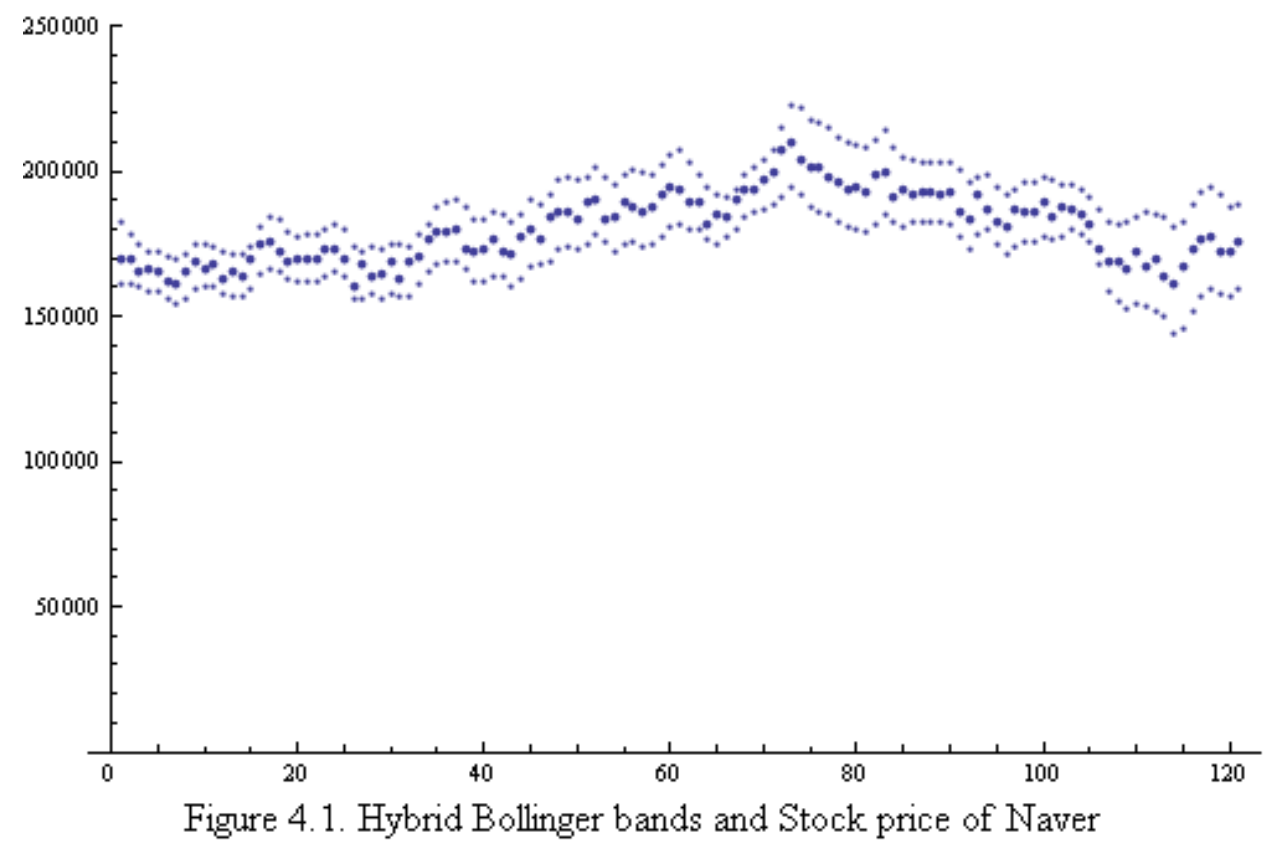

The Naver is a web-based company managing portal site in South Korea. See Figure 4.1. In this graph, we can see that data inside hybrid Bollinger bands is over $95 \%$. This phenomenon is something different from analysis of original Bollinger band. We notify that Figure 4.1 has been rendered by the help of Mathematica Version 7.0.

\section{References}

[B] J. Bollinger, Bollinger on Bollinger Bands, McGraw Hill, New York, 2002.

[C] C.K. Chui, An introduction to wavelets, Academic Press, 1992.

[D] I. Daubechies, Ten Lectures on Wavelets, CBMS-NSF Regional Conference Series in Applied Mathematics, Vol. 61, Society for Industrial and Applied Mathematics, Philadelphia, PA, 1992.

[F] Gepald B. Folland, Real Analysis Modern Techniques and Their Applications, John Wiley \& Sons, 1984, pp. 233-237.

[K] Tom H. Koornwinder, WAVELETS: An Elementary Treatment of Theory and Applications, Series in Approximations and

Decompositions-Vol.1, World Scientific,Singapole,1995. 
[L] J. Lim, Two-Dimensional Signal and Image Processing. Upper Saddle River NJ: Prentice Hall, 1990.

[LHZ] Wei Liu, Xudong Huang, Weian Zhang, Black-Scholes' model and Bollinger bands, Physica A, 371, 2006, pp 565-571.

[O] Alan V. Oppenheim and Alan S. Willsky with S. Hamid Nawab, Signals \& Systems. Prentice-Hall International,INC, 1997.

[R] J. Rhee, Gibbs Phenomenon and certain nonharmonic Fourier series, Communications of Korean Mathematical Society, 2010,

Preprint(to appear).

[St] Elias M. Stein, Harmonic Analysis: Real-Variable Methods, Orthogonality, and Oscillatory Integrals, Princeton University Press, Princeton, New Jersey, 1993, pp. 26-28.

Department of Mathematics

Pusan University of Foreign Studies,

Pusan 608-738, Korea

e-mail : rhee pufs.ac.kr 